\title{
THE POSSIBILITIES OF MR TRACTOGRAPHY IN DIAGNOSTICS OF SYMPTOMATIC EPILEPSY IN CHILDREN WITH DIFFERENT GENESIS
}

\author{
Khabibulla Aminov \\ Tashkent Institute for Postgraduate Medical Education, Tashkent, Uzbekistan
}

\begin{abstract}
In this article the possibilities of MR tractography in symptomatic epilepsy patients have been presented. We determined the quantitative standards of numerical values of diffusion of white matter in children with symptomatic epilepsy of various etiology. In children with symptomatic epilepsy, the pathological changes of white matter were localized not only in the epileptogenic, but also in the opposite hemisphere. The regions with abnormal white matter FA and ADC values not only matched to limbic circle zones, but also to areas of intra- and interhemispheric connections uniting the frontal, temporal, parietal and occipital lobes of the brain. Determining the specific clusters of brain tracts in symptomatic epilepsy, regardless of etiology, is important to predict the prognosis of the disease and the likely possibility of cognitive impairment. The study proved that symptomatic epilepsy is characterized by a variety of changes in the white matter of the brain tracts that lead to the dissociation of certain regions of the brain, interrupting connections between cortical and subcortical regions and inhibiting the transfer of information. It can be concluded, that diffusion-weighted imaging are much more sensitive to the structural and metabolic changes occurring in the brain, in comparison with conventional MRI images.
\end{abstract}

\section{UDC CODE \& KEYWORDS}

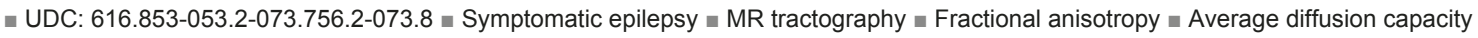

\section{INTRODUCTION}

Research of autoimmune response impacts (formation of antibodies to certain proper phospholipids) on implantation, growth, fetal and fetus devel women $(n=10)$ with physiologically proceeding pregnancy and without APS at the same terms of gestation. Groups were comparable by age, number of gestation and childbirth in past history. Determination of lupus anticoagulant (LAion of D-dimer and FMSC levels is the reflection of haemostasis system since such factors in APS and thrombophilia are elevating long before estimated pregnancy and markers of such processes. In vivo visualization of structural morphological changes of the brain in patients with epilepsy and epileptic syndromes, has been made possible due to the introduction into daily medical practice of neuroradiologist diagnostic methods of research such as: computed tomography and magnetic resonance imaging (CT and MRI) that are classified as "structural" neuroimaging techniques (Gromov, 2003). The introduction of MRI in clinical practice has greatly expanded the identification of various structural changes in the brain tissue underlying the formation of the epileptic focus, which usually leads to the subsequent development of epilepsy. MRI allows radiologists to identify of malformations such as licencephaly, periventricular heterotopy, shizencephaly, a focal cortical dysplasia and other brain anomalies (Bazilevich, Odinak, Diskin, \& Krasakov, 2008). But epilepsy do not always manifest on the background of congenital abnormalities of the brain, particularly if they are not localized in the cortical regions of brain. Congenital disorders of brain has a major part in epilepsy origin, which manifest themselves in early periods of childhood (Thomsen, Henriksen, \& Ring, 1997).

However, in practical medicine, it is not always possible to determine what is the basis of locally-induced epilepsy. Conventional MRI provides information about structural changes not in all cases (Kisten, 2012). In this regard, at modern neuroradiology, it became necessary to use functional neuroimaging techniques that allow not only in vivo study the pathological changes of different brain structures, but also capable to clarify some of the mechanisms of epileptogenesis. These methods include diffusion tensor MRI tractography, which is the technology of the future to obtain morphological images of brain.

Tractography of the brain a diagnostic method based on the diffusion-weighted magnetic resonance imaging (DW MRI), which allows to visualize the orientation and integrity of the pathways of the brain in vivo (Kisten, 2012). Diffusion tensor imaging (DTI) - the technique of magnetic resonance imaging, which allows to estimate the diffusion of water molecules along the myelin sheath of axons of nerve cells in the brain and thus obtain information about the integration of the structures of white matter and the connections between them. Diffusion imaging is an MRI method that produces in vivo magnetic resonance images of biological tissues sensitized with the local characteristics of molecular diffusion, generally water (but other molecules can also be investigated using MR spectroscopic approaches) (Posse, Cuenod, \& Le Bihan, 1993). Using techniques DTI can create a three-dimensional model of neuronal brain. Three-dimensional fiber tractography is a promising new technique that allows to visualize the bundles of nerve fibers that connect different areas of the brain, the link between the axons of the white matter of the brain. MR tractography can more accurately identify the lesions functionally important pathways and improve the quality of diagnosis of congenital diseases of the brain. DTI also improves the evaluation of hypoxic-ischemic lesion of the developing brain of newborns and young children. Interpretation of inter, peri- and postictal diffusion changes is quite complex and difficult, but it has the potential for understanding the mechanisms of undiscovered epileptic seizure (Kisten, 2012). Diffusion-weighted images are much more sensitive to the structural and metabolic changes occurring in the brain, in comparison with standard MRI images. The most important parameter of DT MRI, characterized by the integration of the white matter is fractional anisotropy (FA), which is defined as the magnitude of the direction of water diffusion in three-dimensional-space. Tightly arranged bundles of white matter provide structural coherence, whereby 
THE POSSIBILITIES OF MR TRACTOGRAPHY IN DIAGNOSTICS OF SYMPTOMATIC EPILEPSY IN CHILDREN WITH DIFFERENT GENESIS

the diffusion of water has a definite direction, and FA is a high enough. In case the structural organization of the white matter decreasing and diffusion of water is less orderly manner, the values of FA subsequently diminishes (Le Bihan, 2006). Average diffusion capacity (ADC) is used as indicator to evaluate the results of diffusion tensor MRI.

When the values of ADC are increasing it can be associated with a defect in neurogenesis or cell loss of brain with a consequent increase of the extracellular space of brain (Hagmann et all, 2006).

The aim of this study was to determine the quantitative standards of numerical values of diffusion of white matter in children with symptomatic epilepsy of various etiology.

\section{Materials and Methods}

MRI studies were performed in 31 children with symptomatic epilepsy. Their age ranged from 2 to 12 years. Debut of epileptic seizures was observed from birth to 13 years, mean age debut $4,2 \pm 0,96$ years. Disease duration averaged $3,2 \pm 0,47$ years. The patients were examined and treated at the Department of Paediatric Neurology of Tashkent Paediatric Medical Institute and underwent diagnostic procedures in "StarMed" diagnostic centre from 2012 to 2014. Among the etiological factors of symptomatic epilepsy 11 children had cerebral palsy, 10 children had outcomes of meningoencephalites and 10 children had anomalies of brain. MRI studies were performed on a Philips tomography with a magnetic field of 1.5 T using a head radio frequency coil consisting of 18 elements. The study protocol included a standardized program of MRI of the brain, as well as aiming neuroimaging the mediobasal temporal lobe with thin sections with the possibility of post processing and imaging in different planes. Among the special programs used fast spin-echo T2-weighted, perpendicular to the longitudinal axis of the hippocampus slice thickness of $2 \mathrm{~mm}$, and 3D T1 SP6R - pulse sequences with the reform and obtaining images mediobasal temporal lobe perpendicular and parallel to the longitudinal axis of the hippocampus.

Measurement of anisotropy and diffusivity. Nowadays in clinical neurology, various brain pathologies may be best detected by studying at particular measures of anisotropy and diffusivity. The underlying physical process of diffusion (by Brownian motion) causes a group of water molecules to move out from a central point, and gradually reach the surface of an ellipsoid if the medium is anisotropic (it would be the surface of a sphere for an isotropic medium). The ellipsoid formalism functions also as a mathematical method of organizing tensor data. Measurement of an ellipsoid tensor further permits a retrospective analysis, to gather information about the process of diffusion in each voxel of the tissue (Le Bihan, 2012). In an isotropic medium such as cerebro-spinal fluid, water molecules are moving due to diffusion and they move at equal rates in all directions. By knowing the detailed effects of diffusion gradients we can generate a formula that allows us to convert the signal attenuation of an MRI voxel into a numerical measure of diffusion - the diffusion coefficient $D$. When various barriers and restricting factors such as cell membranes and microtubules interfere with the free diffusion, we are measuring an "apparent diffusion coefficient" or ADC because the measurement misses all the local effects and treats it as if all the movement rates were solely due to Brownian motion (Basser, Mattiello \& Le Bihan, 1994). The ADC in anisotropic tissue varies depending on the direction in which it is measured. Diffusion is fast along the length of (parallel to) an axon, and slower perpendicularly across it. Once we have measured the voxel from six or more directions and corrected for attenuations due to T2 and T1 effects, we can use information from our calculated ellipsoid tensor to describe what is happening in the voxel. If you consider an ellipsoid sitting at an angle in a Cartesian grid then you can consider the projection of that ellipse onto the three axes. The three projections can give you the $A D C$ along each of the three axes $A D C_{x}, A D C_{y}$, $A D C_{z}$. This leads to the idea of describing the average diffusivity in the voxel which will simply be

$$
\left(A D C_{x}+A D C_{y}+A D C_{z}\right) / 3=A D C_{i}
$$

We use the i subscript to signify that this is what the isotropic diffusion coefficient would be with the effects of anisotropy averaged out. The ellipsoid itself has a principal long axis and then two more small axes that describe its width and depth. All three of these are perpendicular to each other and cross at the centre point of the ellipsoid. We call the axes in this setting eigen vectors and the measures of their lengths eigen values. The lengths are symbolized by the Greek letter $\lambda$. The long one pointing along the axon direction will be $\lambda_{1}$ and the two small axes will have lengths $\lambda_{2}$ and $\lambda_{3}$. In the setting of the DTI tensor ellipsoid, we can consider each of these as a measure of the diffusivity along each of the three primary axes of the ellipsoid. This is a little different from the ADC since that was a projection on the axis, while $\lambda$ is an actual measurement of the ellipsoid we have calculated. The diffusivity along the principal axis, $\lambda_{1}$ is also called the longitudinal diffusivity or the axial diffusivity or even the parallel diffusivity $\lambda_{\|}$. Historically, this is closest to what Richards originally measured with the vector length in 1991 (Richards,1992). The diffusivities in the two minor axes are often averaged to produce a measure of radial diffusivity.

$$
\lambda_{\perp}=\left(\lambda_{2}+\lambda_{3}\right) / 2
$$

This quantity is an assessment of the degree of restriction due to membranes and other effects and proves to be a sensitive measure of degenerative pathology in some neurological conditions. It can also be called the perpendicular diffusivity $(\lambda \perp)$. Another commonly used measure that summarizes the total diffusivity is the Trace-which is the sum of the three eigen values,

$\operatorname{tr}(\Lambda)=\lambda_{1}+\lambda_{2}+\lambda_{3}$ where $\Lambda$ is a diagonal matrix with eigen values, and on its diagonal. If we divide this sum by three we have the mean diffusivity,

$$
M D=\left(\lambda_{1}+\lambda_{2}+\lambda_{3}\right) / 3
$$

which equals $\mathrm{ADC}_{\mathrm{i}}$ since,

$$
\begin{aligned}
\operatorname{tr}(\Lambda) / 3 & =\operatorname{tr}\left(V V^{-1} \Lambda\right) / 3 \\
& =\operatorname{tr}\left(V \wedge V^{-1}\right) / 3 \\
& =\operatorname{tr}(D) / 3 \\
& =A D C_{i}
\end{aligned}
$$


THE POSSIBILITIES OF MR TRACTOGRAPHY IN DIAGNOSTICS OF SYMPTOMATIC EPILEPSY IN CHILDREN WITH DIFFERENT GENESIS

Diffusion tensor is calculated for each voxel, resulting in the received image (map) the fractional anisotropy and the average diffusivity. Tractography was performed for all examined with the assessment of the number of paths of lines for front and rear quadrant in axial sections. The values of FA and ADC were calculated on the same sections for all the resulting images. The data obtained were subjected to statistical processing on a PC Pentium-4 program, developed in the package EXCEL using a library of statistical functions with the calculation of the arithmetic mean (M), standard deviation (SD), standard error (SE), relative values (frequency, \%), Student's $t$ test $(t)$ with the computation of error probability (P). Differences were considered significant mean values at a significance level of $P<0.05$.

\section{Results of the Study}

In a study of 11 children with epilepsy on the background of symptomatic epilepsy with cerebral palsy diffusion tensor MRI tractography allowed to determine the micro structural changes in 9 children. The most important indicator of the integration of the white matter of the brain is the value of the FA (Hagmann et all, 2012). This indicator is in group of children with cerebral palsy was $0,54 \pm 0,006(0,52 \div 0,56)$ for the front quadrants and $0,55 \pm 0,006(0,52 \div 0,57)$ for the rear quadrants. Hemispheric asymmetry in this parameter revealed only for the rear quadrant, the main area which represented the temporal lobes, with preferential reduction of FA was determined in the hemisphere with epileptic focus $(p<0.05)($ Table. 1).

Table 1: Indicators of FA and ADC on MRI tractography in children with symptomatic epilepsy with various etiology

\begin{tabular}{|l|l|l|l|l|}
\hline \multirow{2}{*}{ Etiology of symptomatic epilepsy } & Indicators of FA & \multicolumn{2}{l|}{ Indicators of ADC } \\
\cline { 2 - 5 } & Front quadrants & Rear quadrants & Front quadrants & Rear quadrants \\
\hline Normal brain & 0,56 & 0,57 & 0,83 & 0,85 \\
\hline Cerebral Palsy & $0,54 \pm 0,006^{*}$ & $0,55 \pm 0,006^{*}$ & $0,84 \pm 0,005$ & $0,86 \pm 0,006$ \\
\hline After meningoencephalites & $0,55 \pm 0,006$ & $0,55 \pm 0,009$ & $0,86 \pm 0,007^{*}$ & $0,87 \pm 0,004^{*}$ \\
\hline Brain anomalies & $0,48 \pm 0,03^{* *}$ & $0,47 \pm 0,02^{* *}$ & $1,01 \pm 0,33^{* *}$ & $0,87 \pm 0,03$ \\
\hline Note: ${ }^{*}$ - the accuracy of the data compared with the norm $\left(^{*}-\mathrm{P}<0.05 ;{ }^{* *}-\mathrm{P}<0.01\right)$ \\
\hline
\end{tabular}

Source: Author

At the same time 10 children had the lowest level of FA for the front quadrants, with deviation of $3.7 \%$. In 8 children observed deviations of FA in rear quadrants with $4.3 \%$. The values of ADC have been counted to study the mechanisms of micro structural changes in brain. ADC values in $54.5 \%$ of children with symptomatic epilepsy on the background of cerebral palsy differed from normal values for the front quadrant of the hemisphere with the epileptic focus and posterior regions of the cerebral hemispheres $(P<0.05)$. While observing 11 children with symptomatic epilepsy in 6 children $A D C$ value for front quadrant of the hemisphere was increased by an average of $1.53 \%$ then normal values, and for the rear quadrant of the hemisphere on average by $1.1 \%$ of normal values (Fig. 1).

Figure 1: The percentage of abnormalities in FA values in children with symptomatic epilepsy, depending on the etiology (\%)

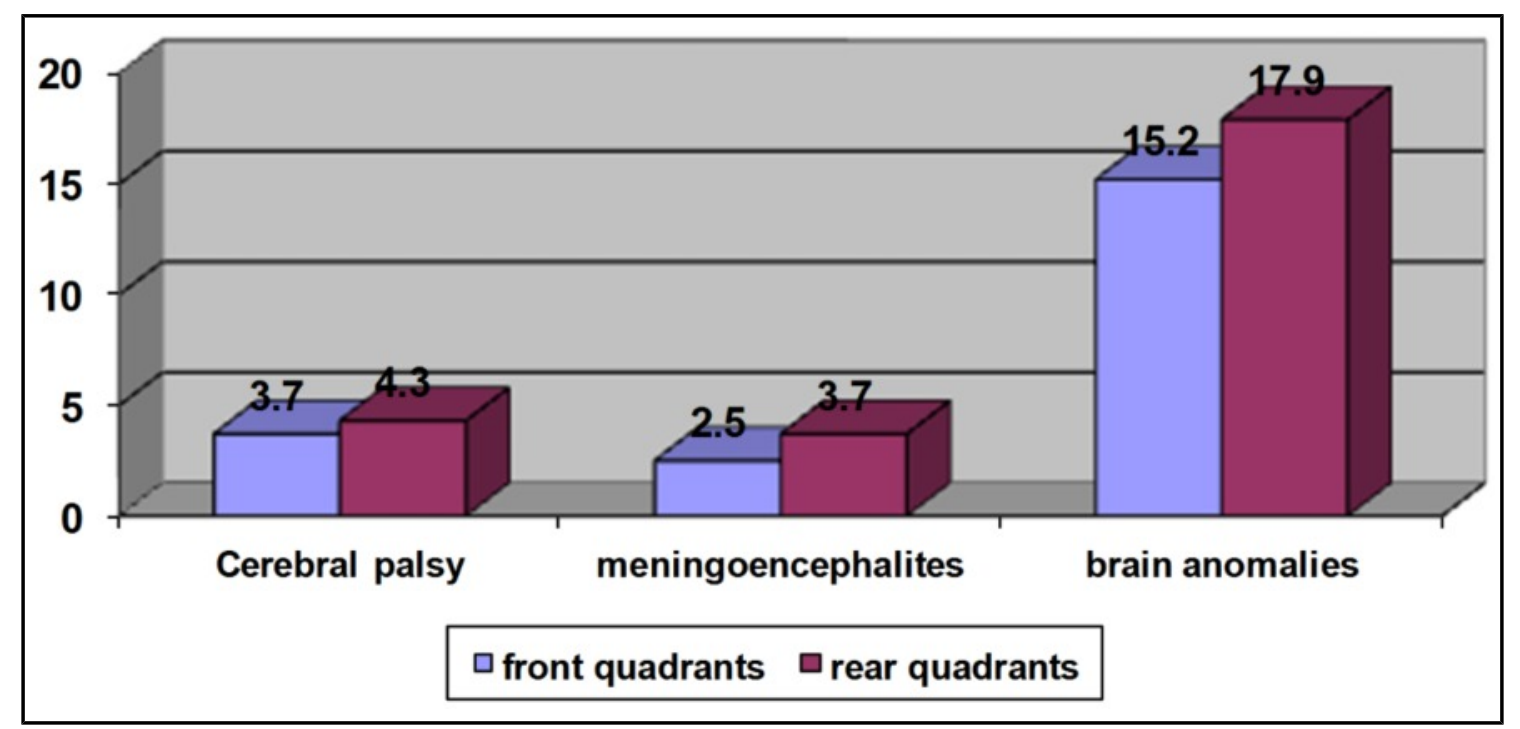

Source: Author (2014)

Thus, in children with symptomatic epilepsy on a background of cerebral palsy in most cases there was a decrease of FA for both front and rear quadrant on the background of increasing ADC.

Among the 10 children with symptomatic epilepsy after meningoencephalitis FA values was different, because the average number of FA values were $0,55 \pm 0,006$ for the front quadrants and $0,55 \pm 0,009$ for the rear ones. Indicators of $A D C$ for front quadrants were $0,86 \pm 0,007$ and for the rear ones were $0,87 \pm 0,004$. Deviations from normal values to down on average in FA was $2.5 \%$ for front quadrants, for the rear was $3.7 \%$. When analyzing ADC values different pattern was revealed, so the mean deviation from the normal values to upwards in front quadrants was $3.6 \%$, for the rear ones was $2.6 \%$ (Fig.2).

http://www.journals.cz 


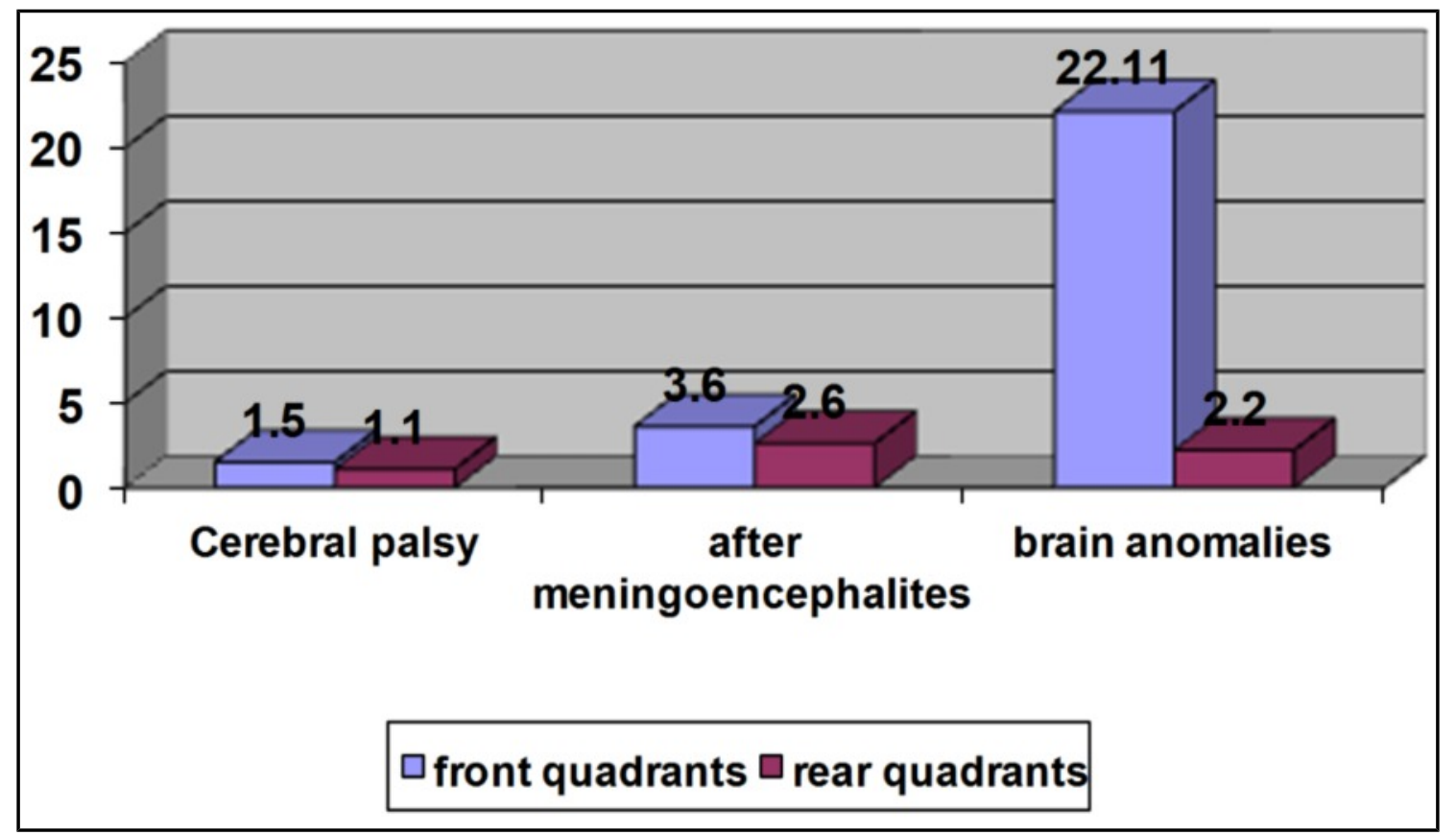

Source: Author (2014)

Analyzing the data of MRI tractography in children with symptomatic epilepsy on the background abnormalities of brain development we have identified low levels of FA for both front and rear quadrants $(0,48 \pm 0,03$ and $0,47 \pm 0,02$, respectively; $\mathrm{P}<0.05$ ) as compared with the normal values. A deviation from the normal FA had $15.2 \%$ for the front quadrants and $17.9 \%$ for the rear. Indicators of ADC in children with symptomatic epilepsy on a background of developmental abnormalities of the brain underwent the following changes: the front quadrants figures were 1,01 $\pm 0,33(p<0.05)$ for the rear $-0,87 \pm 0,03$ $(P<0.05)$. There was a significant deviation of ADC from normal values, $22.11 \%$ for the front quadrants and $2.2 \%$ for the rear. Thus, in children with symptomatic epilepsy on a background of developmental abnormalities of the brain there was a sharp decline of the FA for front and rear quadrants and a huge increase of the ADC for the front quadrants. According to the results of the studied children following four clusters were identified:

1. reduction of representation tracts in the frontal regions of the brain;

2. reduced representation paths in the parietal-temporal areas of the cerebral hemispheres;

3. reduction of the representation front and rear commissure;

4. asymmetry tracts of the cerebral hemispheres.

In children with symptomatic epilepsy on the background of cerebral palsy in most cases first, second, third clusters were detected $(7,10$ and 6 children, respectively). The combination of these clusters observed in 8 cases $(72.7 \%)$. In children with symptomatic epilepsy on the background of meningoencephalites in most cases, there was a first and second cluster ( 7 and 10 children, respectively). The combination of all four clusters were recorded in $90 \%$ of children. Changes in the state of integrity of the corpus callosum was observed among $54.5 \%$ of children with cerebral palsy, and $50 \%$ of children with developmental abnormalities of the brain appeared as a hypoplasia or hypogenesia. According to other authors state integrity of the corpus callosum provides stability of cognitive and emotional status of the children. Thus, the presence of these clusters to some extent indicates the "interest" of the various regions of the brain in promoting epileptic seizures, and is being as predictor of the developing of the disease.

\section{CONCLUSION}

We revealed that in children with symptomatic epilepsy, regardless of its etiology, the presence of discrete regions of the abnormal changes of the white matter of the brain was discovered, while the pathological changes of white matter were localized not only in the epileptogenic, but also in the opposite hemisphere. The regions with abnormal white matter FA and ADC values not only matched to zones of limbic circle, but also to areas of intra- and interhemispheric connections uniting the frontal, temporal, parietal and occipital lobes of the brain. Determining of specific clusters of lesions of the brain tracts in symptomatic epilepsy in children, regardless of etiology, is important to predict the prognosis of the disease and the likely possibility of cognitive impairment. Status integrity of the corpus callosum provides stability of cognitive and emotional status of children (Wedeen et all., 2008). Thus, this study showed that symptomatic epilepsy in children is characterized by a variety of changes in the white matter of the brain tracts that lead to the dissociation of certain regions of the brain, which subsequently causes further breaking connections between cortical and subcortical regions and interrupting transfer of information. MRI tractography allows to extend the representation of micro structural changes in integrity of gray and white matter of brain in symptomatic epilepsy and clarify the structural and metabolic etiologic subtype of the disease according to the recommendations of the International League Against Epilepsy. 


\section{REFERENCES}

Basser P.J, Mattiello J, \& Le Bihan D (1994). MR diffusion tensor spectroscopy and imaging. Biophysical Journal 66 (1): $259-267$. Bibcode:1994BpJ...66..259B. DOI: 10.1016/S0006-3495(94)80775-1. PMC 1275686. PMID 8130344

Bazilevich, C.N., Odinak, M.M., Diskin, D.E., \& Krasakov, I. V. (2008). The results of structural and functional neuroimaging in patients with epileptic seizures in cerebrovascular diseases. Journal of neurology named after S.Korsakov (Epilepsy. Appendix of the journal). 2: 33-39.

Gromov, S.A. (2003). Epilepsy: proceedings of the conference "Actual problems of modern neurology, psychiatry and neurosurgery." 21-26.

Hagmann, B., Jonasson, L., Maeder, P., Thiran, J., Pandya, D., \& Meuli, R. Understanding Diffusion MR Imaging Techniques: From Scalar Diffusion-weighted Imaging to Diffusion Tensor Imaging and Beyond. RadioGraphics. Oct $2006 . \quad$ Retrieved from http://radiographics.rsna.org/content/26/suppl_1/S205.full

Kisten, O.V. (2012). Experience of diffusion tensor magnetic resonance imaging in the morphological diagnosis of epilepsy. Neurology and Neurosurgery. 4: 95-97.

Le Bihan D, (2012) . MR imaging of intravoxel incoherent motions: application to diffusion and perfusion in neurologic disorders. Radiology, 161: $401-407$

Le Bihan, D. (2006). Direct and fast detection of neuronal activation in the human brain with diffusion MRI. Proceedings of the National Academy of Sciences 103 (21): 8263-8268. Bibcode: 2006PNAS..103.8263L. DOI: 10.1073/pnas.0600644103.

Posse, S., Cuenod, C.A., \& Le Bihan, D. (1993). Human brain: proton diffusion MR spectroscopy. Radiology 188 (3): $719-25$. PMID 8351339.

Richards, T.L., Heide, A.C., Tsuruda, J.S., \& Alvord, E.C. (1992). Vector analysis of diffusion images in experimental allergic encephalomyelitis. Presented at Society for Magnetic Resonance in Medicine, Berlin, SMRM Proceedings 11: 412, (abstr).

Thomsen, C., Henriksen, O., \& Ring, P. (1997). In vivo measurement of water self diffusion in the human brain by magnetic resonance imaging. Acta Radiologica, 28: 353-361.

Wedeen, V.J. Wang, R.P. Schmahmann, J.D., Benner, T., Tseng, W.Y, Dai, G., Pandya, D.N., Hagmann, P., \& D'arceuil, H. (2008). Diffusion spectrum magnetic resonance imaging (DSI) tractography of crossing fibers. Neurolmage 41 (4): 1267-77. DOI: 10.1016/j.neuroimage.2008.03.036. PMID 18495497. 